\title{
Expression of Fatty Acid Synthase Genes and their Role in Development and Arboviral Infection of Aedes Aegypti
}

\author{
Nunya Chotiwan \\ Colorado State University \\ Carlos A. Brito-Sierra \\ Purdue University \\ Gabriella Ramirez \\ Colorado State University \\ Elena Lian \\ Colorado State University \\ Catherine A. Hill \\ Colorado State University \\ Rushika Perera ( $\sim$ rushika.perera@colostate.edu ) \\ Colorado State University https://orcid.org/0000-0001-6798-2537
}

\section{Research Article}

Keywords: Aedes aegypti, Aag2 cells, fatty acid synthase, FAS, lipid, lipid metabolism, dengue virus, AaegL5 genome assembly

Posted Date: February 16th, 2022

DOI: https://doi.org/10.21203/rs.3.rs-1340331/v1

License: (c) (1) This work is licensed under a Creative Commons Attribution 4.0 International License. Read Full License 


\section{Abstract \\ Background}

Fatty acids are the building blocks of complex lipids essential for living organisms. In mosquitoes, fatty acids are involved in cell membrane production, energy conservation and expenditure, innate immunity, development, and reproduction. Fatty acids are synthesized by a multifunctional enzyme complex called fatty acid synthase (FAS). Several paralogues of FAS were found in the Aedes aegypti (Ae. aegypti) mosquito. However, the molecular characteristics and the expression of some of these paralogues have not been investigated.

\section{Methods}

Genome assemblies of Ae. aegypti were analyzed and orthologues of human FAS were identified. Phylogenetic analysis and in silico molecular characterization were performed to identify the functional domains of the Ae. aegypti FAS (AaFAS). Quantitative analysis and loss-of-function experiments were performed to determine the significance of different AaFAS transcripts in various stages of development, expression following different diets and the impact of $A a F A S$ on dengue virus, serotype 2 (DENV2) infection and transmission.

\section{Results}

We identified seven putative FAS genes in the Ae. aegypti genome assembly, based on nucleotide similarity to the FAS proteins (tBLASTn) of humans, other mosquitoes and invertebrates. Bioinformatics and molecular analyses suggested that only five of the FAS genes produce mRNA and therefore represent complete gene models. Expression levels of AaFAS varied among developmental stages and between male and female Ae. aegypti. Quantitative analyses revealed that expression of AaFAS1, the putative orthologue of the human FAS, was highest in adult females. Transient knockdown (KD) of $A a F A S 1$ did not induce a complete compensation by other AaFAS genes but limited DENV2 infection of Aag2 cells in culture and the midgut of the mosquito.

\section{Conclusion}

AaFAS1 is the predominant AaFAS in the adult mosquitoes. It has the highest amino acid similarity to human FAS and contains all enzymatic domains typical of human FAS. AaFAS1 also facilitated DENV2 replication in both cell culture and in mosquito midguts. Our data suggest that $A a F A S 1$ may play a role in transmission of dengue viruses, and could represent a target for intervention strategies.

\section{Background}

Fatty acid synthase (FAS) is a multifunctional enzyme catalyzing more than 40 steps in the de novo fatty acid biosynthesis pathway (1) (2). It contains seven catalytic and three non-catalytic domains which condense, reduce and dehydrate the three-carbon substrate, malonyl-CoA, into 16 to 18 -carbon fatty acids. These fatty acids are essential building blocks of complex lipids, such as phosphoglycerolipids, glycerolipids and sphingolipids, which are components of cellular membranes and storage lipids, and function as signaling molecules, respectively.

In mosquitoes, fatty acids also play roles in innate immunity, reproduction, development, and flight (3-5). Fatty acids can be acquired or synthesized in both larvae and adult stages. Neonate larvae acquire lipids through the maternal deposition in eggs (6-8) and through consumption of aquatic diets such as diatoms and algae, which are the primary source of polyunsaturated fatty acids (9). Fatty acids from larval stages can be transferred to the adult stage and some can be deposited in eggs of the first gonotrophic cycle (10). Adult mosquitoes possess enzymes for de novo synthesis and modification of fatty acids from both sugar (carbohydrate-enriched) and blood (protein-enriched) meals $(7,9,11)$. In the female, fatty acid synthesis is important for metabolism and production of eggs. Transient knockdown (KD) of acetyl-CoA carboxylase (ACC) and fatty acid synthase (FAS), two key enzymes in the de novo fatty acid biosynthesis pathway, led to significantly lower egg production in the first gonotrophic cycle (12). In addition, eggs produced by ACC-KD mosquitoes lacked eggshells and were nonviable (12).

Apart from its importance to mosquito biology, studies suggest FAS also plays a supportive role for several arboviral infections in both mammalian and mosquito cells (13-16). It is known that several RNA viruses induce expansion and rearrangement of host cell membranes to support viral genome replication and assembly (17-19). Studies have shown that FAS facilitates the production of dengue virus serotype 2 (DENV2) infection in both human and mosquito cells, potentially by providing the building blocks for this membrane expansion event $(13,15)$. Lastly, studies also reported the elevation of fatty acid abundance in C6/36 (Aedes albopictus) cells, and in the Aedes aegypti (Ae. aegypti) mosquito midgut during DENV2 infection (15, 20). These findings suggest that fatty acids are essential for the physiological function of mosquitoes, and support DENV2 infection of the mosquito.

Currently, understanding of FAS in mosquitoes and its role in pathogen transmission by the mosquito vector is limited. Here, we describe the molecular and functional characterization of the FAS gene family from Ae. aegypti (AaFAS). We identified seven putative AaFAS genes (AaFAS 1-6 and AaFAS-like) in the AaegL5 genome assembly, characterized the expression of these genes during mosquito development and following consumption of different diets. AaFAS1 had the highest amino acid similarity to human FAS and was the predominant transcript. We investigated the role of AaFAS1 in DENV2 infection in mosquito cells and live mosquitoes using gene KD. We observed a significant reduction of DENV2 replication following AaFAS1-KD in Ae. aegypti cells and a transient reduction of infection in Ae. aegypti midguts at early time points post-infectious blood meal. These results provide insights to the molecular characteristic of AaFASs and their role during Ae. aegypti development, food source acquisition and arbovirus infection. 


\section{Methods}

\section{Alignments, conserved motifs and phylogenetic tree}

Putative AaFAS sequences from the AaegL5 genome assembly were blasted against the AaegL3 genome assembly retrieved from VectorBase using tBLASTn (21-23). FAS sequences of Anopheles gambiae, Drosophila melanogaster, Apis mellifera, Homo sapiens, Mus musculus and Saccharomyces cerevisiae were aligned with putative AaFAS sequences using ClustalW (24). mRNA sequences were retrieved from NCBI and manually curated to confirm the intron/exon boundaries. Conserved FAS motifs were identified by global alignment of vertebrate, invertebrate and yeast proteins using the Clustal Omega and Jalview 2.11.1.5 (accession numbers are shown in Table S1) (25) and conserved amino acids associated with catalytic domains of functional FAS were identified by comparison to sequences reported in published studies (26). Individual amino acid alignments were also performed between FAS-AaegL5 and FAS-AaegL3 using ClustalW to identify improvements in AaegL5 models.

A Bayesian inference of phylogeny was performed using the amino acid sequence of FAS from Ae. aegypti, Anopheles gambiae, Drosophila melanogaster, Apis mellifera, Mus musculus and Homo sapiens. Yeast Kexin was used as an outgroup. A sequence alignment with ClustalW was performed prior to tree construction in phylogeny.fr. The substitution model used for the Bayesian inference was Blosum62 and the Markov Chain Monte Carlo parameters included 100,000 generations with sampling every 10 generations, discarding the first 250 trees. The resulting tree was annotated and curated in iTOL.

Annotation of protein domains in Ae. aegypti FAS genes

AaFAS amino acid sequences were aligned against the human FAS (NP_004095.4, NCBI) using Clustal Omega (27) to identify the seven catalytic and three noncatalytic domains associated with mammalian FAS. The alignment results were viewed using MView tool (28). Motifs in the human FAS were identified based on Pfam 31.0 (29) and conserved domains in AaFAS genes were identified by comparative analyses.

\section{Mosquito rearing}

Larvae and adults of Aedes aegypti strain Chetumal, originally collected from Yucatan Peninsula in Mexico, were reared on fish food and on $10 \%$ sucrose solution, respectively and adults maintained under constant conditions of $28^{\circ} \mathrm{C}, 80 \%$ relative humidity (30).

\section{Blood feeding}

Twenty-four hours prior to blood feeding, mosquitoes were starved for 4 hours by removal of sucrose solution. Defibrinated sheep blood (Colorado Veterinarian Product) was mixed with $1 \mathrm{mM}$ ATP and placed in an artificial membrane feeder warmed by a $37^{\circ} \mathrm{C}$ water jacket. Mosquitoes were allowed to feed for 45-60 minutes. Fully engorged mosquitoes were sorted and reared on $10 \%$ sucrose solution and water.

\section{Generating long double-stranded RNA}

Long double-stranded RNA (dsRNA) was generated from Ae. aegypti mosquito total RNA. Primers were designed to amplify an $~ 500$ bp region of the gene of interest (Table S2). cDNA was generated by reverse transcription (RT) using specific reverse primers and SuperScript III Reverse Transcriptase (Invitrogen). Polymerase chain reaction (PCR) was performed using specific primers containing a $5^{\prime} \mathrm{T} 7$ promotor sequence adapted to both forward and reverse primers and Taq polymerase (NEB). PCR products were purified using the GeneJET PCR Purification kit (Thermo Scientific) and in vitro transcription was performed using the MEGAscript T7 kit (Invitrogen) and incubation at $37^{\circ} \mathrm{C}$ for 12 hours. Following incubation, the product was heated to $75^{\circ} \mathrm{C}$ for 5 minutes and slowly cooled to room temperature for 4 hours to dsRNA annealing. Next, dsRNA was treated with DNase (NEB) and purified by phenol-chloroform extraction followed by ethanol precipitation and the purified dsRNA was stored at $-80^{\circ} \mathrm{C}$.

dsRNA knockdown of AaFAS1 in Ae. aegypti moquitoes

dsRNA was introduced via intrathoracic (IT) injection of adult females at 3 to 4 days post-eclosion (31). Mosquitoes were anesthetized at $4^{\circ} \mathrm{C}$ on a cold plate. Glass needles were prepared with a vertical pipette puller (P-30, Sutter Instrument Co., Novato, CA) and mosquitoes were IT injected with $3 \mu \mathrm{g} / \mu \mathrm{l}$ of dsRNA in an injection volume of $69 \mathrm{nl}$, twice (total of $\sim 400 \mathrm{ng}$ of dsRNA) using a Nanojet II (Drummond Scientific Company, Broomall, PA). Injected mosquitoes were fed on sucrose solution or blood and reared at $28^{\circ} \mathrm{C}, 80 \%$ relative humidity for 17 days post-injection.

\section{dsRNA knockdown of AaFAS1 gene and DENV2 infection of Aag2 cells}

dsRNA KD was performed in RNA interference-competent Ae. aegypti (Aag2) cells. Aag2 cells were cultured in Schneider's insect medium (Sigma-Aldrich) supplemented with $2 \mathrm{mM} \mathrm{L-glutamine,} 1 \%$ non-essential amino acids and 10\% FBS. The cells were seeded in a 48-well plate at 50,000 cells/well for 24 hours, and subsequently transfected with $260 \mathrm{ng}$ of dsRNA mixed with TransIT-2020 Reagent (Mirus) following the manufacturer's protocol. New medium with $2 \%$ FBS was replaced at 6 hours post-transfection. Cell viability assays were performed at 2 days post-transfection using resazurin assay.

KD cells were infected with infectious DENV2 expressing a luciferase reporter (DEN-Luc) supplied by C. Rice, Rockefeller University. Cell culture medium was replaced with $300 \mu \mathrm{l}$ of DEN-Luc supernatant at 48 hours post dsRNA transfection, and cells were incubated at $28^{\circ} \mathrm{C}$ without $\mathrm{CO}_{2}$. Virus supernatant was removed at 24 hours post-infection, and cells were lysed, and luciferase activity was read using the Luciferase Assay System (Promega) as per manufacturer protocol.

\section{Gene expression analyses}

Total RNA was extracted from dissected midgut or whole mosquito by TRIzol (Life Tech) and cDNA was produced via reverse transcription using random primers (Life Tech) and SuperScript III Reverse Transcriptase (Invitrogen). Approximately $400 \mathrm{ng}$ of total cDNA was employed for quantitative PCR (qPCR) 
analyses. Gene-specific primers are listed in Table S3. $\beta$-actin was used as a reference gene. Relative AaFAS gene expression was assessed by normalization to the levels of the $\beta$-actin gene $\left(2^{-\Delta C t}\right)$. The comparative $\mathrm{Ct}\left(2^{-\Delta \Delta C t}\right)$ method was used to calculate the relative expression of $A a F A S$ following treatment compared to the control (32).

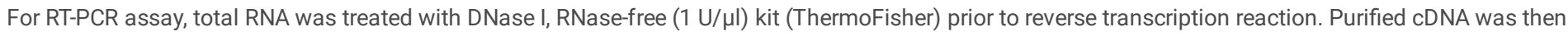
amplified using Q5 ${ }^{\circledR}$ High-Fidelity DNA Polymerase kit (New England BioLabs) with following condition, $98^{\circ} \mathrm{C}$ for 30 seconds, 35 cycles of $98^{\circ} \mathrm{C}$ for 10 seconds, $68^{\circ} \mathrm{C}$ and $72^{\circ} \mathrm{C}$ for 2 minutes and 30 seconds. primers are listed in Table S4.

\section{Virus infection of Ae. aegypti by infectious blood meal}

DENV2 serotype 2 strain Jamaica-1409 (33) was cultured in C6/36 cells. Cells were infected with DENV2 at a multiplicity of infection of 0.01 and incubation at room temperature for 1 hour. Virus supernatant was removed, and infected cells were cultured in $5 \mathrm{ml}$ total volume of L15 medium supplemented with $3 \%$ fetal bovine serum (FBS), $50 \mu \mathrm{g} / \mathrm{ml}$ penicillin-streptomycin, and $2 \mathrm{mM} \mathrm{L-glutamine.} \mathrm{Media} \mathrm{was} \mathrm{replaced} \mathrm{at} 7$ days post-infection (dpi) and virus supernatant was harvested on 12-14 dpi and immediately used to prepare the infectious blood meal.

\section{Midgut dissection and plaque titration}

Mosquito tissues were collected at multiple days post-exposure to the virus indicated in the figure legends. Isolated midguts or the mosquito carcass (remainder of the body without midgut) were placed separately into $2 \mathrm{ml} \mathrm{safe-lock} \mathrm{Eppendorf} \mathrm{tubes} \mathrm{(Eppendorf)} \mathrm{containing} 250 \mu \mathrm{l}$ of mosquito diluent ( $1 \times$ PBS supplemented with $20 \%$ FBS, $50 \mu \mathrm{g} / \mathrm{ml}$ Penicillin/Streptomycin (Gibco), $50 \mu \mathrm{g} / \mathrm{ml}$ Gentamycin (Gibco), and $2.5 \mu \mathrm{g} / \mathrm{ml}$ Amphotericin B (Gibco)) and a stainless-steel bead (34). Tissue was homogenized using a Retsch Mixer Mill MM400 at 24 cycles per second for 1 minute, centrifuged at $15,000 \mathrm{~g}$ for 5 minutes at $4^{\circ} \mathrm{C}$ and supernatant was transferred to a new tube for plaque titration.

Plaque assay was performed on BHK-15 cells. Ten-fold serially diluted viral supernatant was absorbed on the confluent cell layer. After 45 minutes of absorption, cells were overlaid with 1x Minimum Essential Media (MEM), 1X agar supplemented with 2.5\% FBS, $25 \mu \mathrm{g} / \mathrm{ml} \mathrm{Penicillin/Streptomycin,} 25 \mu \mathrm{g} / \mathrm{ml}$ Gentamycin, and $1.25 \mu \mathrm{g} / \mathrm{ml}$ Amphotericin B and the cells were incubated at $37^{\circ} \mathrm{C}$ with $5 \% \mathrm{CO}_{2}$. Cells were stained with $0.033 \%$ neutral red (Sigma) in $1 \times$ PBS on day 5 post-infection and plaques were counted at 24 hour post-staining.

\section{Results}

\section{Molecular Characterization of Ae. aegypti FAS genes}

Seven putative AaFAS genes models were obtained via manual annotation using the AaegL5 assembly (35). Previously, five candidate FAS genes (AaFAS1-5), were identified based on the AaegL3 assembly of Nene et al., 2007 (23, 36), and of these, only AaFAS1 and 2 have undergone functional studies (12). The AaegL5 assembly enabled identification of two additional candidate FAS genes (AaFAS6 and AaFAS-like). The corresponding mRNA sequences showing predicted intron/exon structure and initiation and stop codons are shown in Supplemental File 1. The AaFAS1 gene model revealed a gene structure comprising 11 exons, while AaFAS2 had 5 exons and AaFAS3-5 had 6 exons (Fig. 1). The incomplete AaFAS-like and AaFAS6 gene models comprised 2 and 3 exons, respectively.

The gene models for AaFAS1-5 appear to be full length, with an average gene product length of 2,360 amino acids (Table 1). AaFAS1-5 possessed features associated with functional FAS, including an initiation methionine, a stop codon and the functional catalytic motifs (DTACSS, EAH and GSVKS) important for ketoacyl synthesis as described by Beedessee et al. 2015 (26). Additionally, AaFAS1-5 contained the YKELRLRGY motif conserved among the FAS genes of vertebrates and invertebrates, present in the polyketide synthase deshydratase domain (Fig. S1). AaFAS3 lacked 6 amino acid residues in the 3 ' terminus of exon 6 and a total of 127 non-synonymous substitutions were identified in this model as compared to its AaegL3 counterpart.

Table 1. Summary of AaFAS gene family predicted from the Aedes aegypti AaegL5 assembly. The AaegL5 annotation is shown in comparison to the AaegL3 gene models reported by Nene et al., 2007 [23]. 


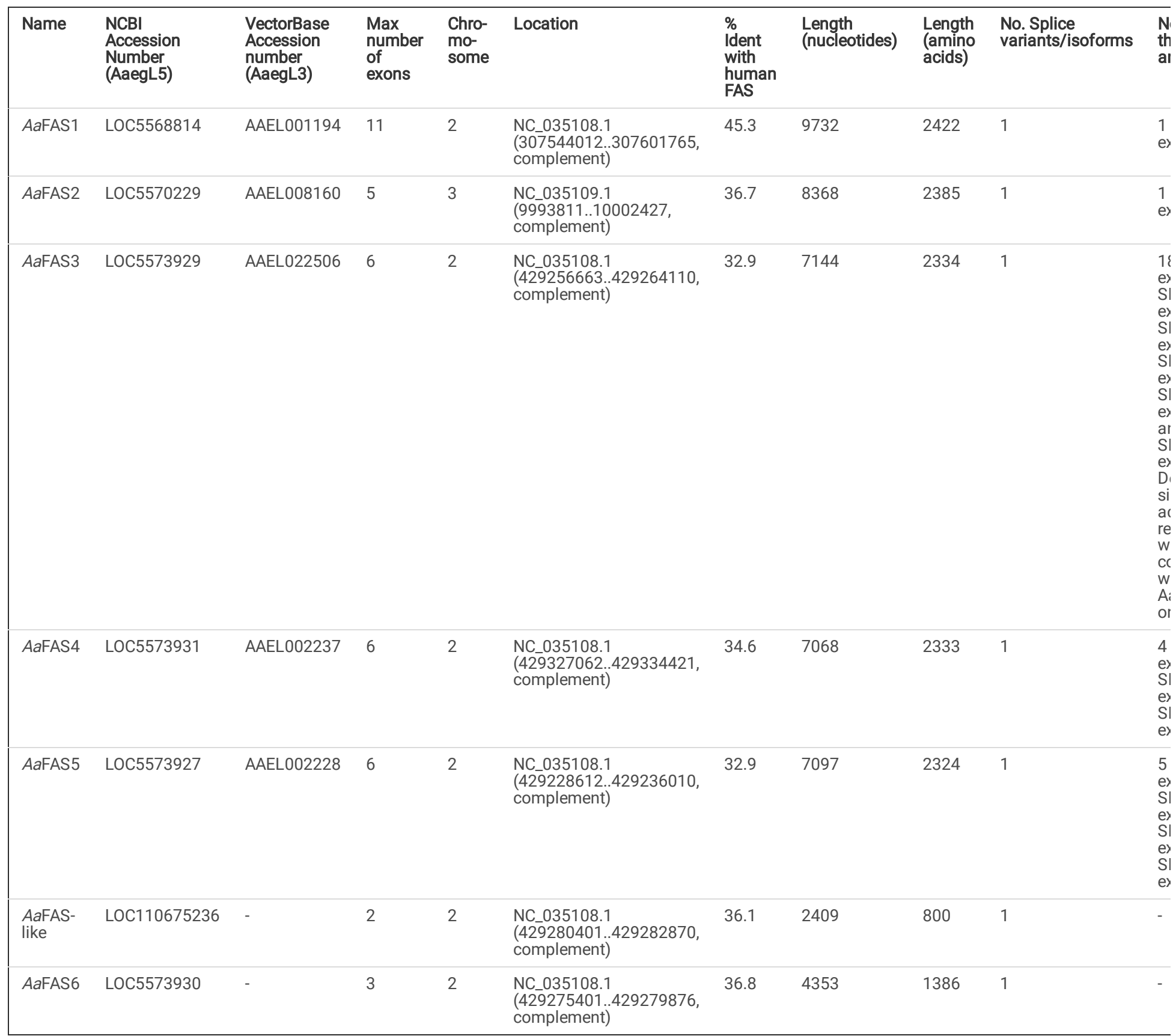

The Bayesian inference supported AaFAS1-5 as paralogues, and revealed highest percent amino acid similarity between AaFAS1 and the $H$. sapiens FAS (human FAS) (Fig. 2). Notably, AaFAS1 clustered in a clade comprising the H. sapiens, Mus musculus, Apis mellifera FAS, the D. melanogaster FAS1 and 2, and an uncharacterized Anopheles gambie FAS (Fig. 2). Similarly, AaFAS2 clustered in a clade with another uncharacterized Anopheles gambiae FAS. In contrast, AaFAS3, 4, 5, 6 and -like clustered at the most branched portion of the tree, suggesting a recent diversification event. Phylogenetic analyses and amino acid alignment supported AaFAS1-5 as the counterparts of the AaegL3 genome assembly-derived gene models as follows: LOC5568814-AAEL001194; LOC5570229-AAEL008160; LOC5573929-AAEL022506; LOC5573931-AAEL002237 \& LOC5573927-AAEL002228 (Fig. 2, Table 1). AaFAS-like and AaFAS6 (LOC110675236 \& LOC5573930) were not identified in the AaegL3 assembly suggesting these models are unique to the AaegL5 assembly.

To investigate putative functional domains, AaFAS sequences were aligned to the human FAS using Clustal Omega (27). Human FAS contains seven catalytic domains and three noncatalytic domains (1). Collectively, AaFAS posssesed less than $50 \%$ amino acid identity to human FAS, and of the seven gene models, AaFAS1 had the highest amino acid identity (45.3\%) (Table 1 and Table S5). Alignment of FAS domains also showed modest sequence identity between human FAS and AaFAS (23.03-63.56\%) with greatest similarity for AaFAS1 domains (Table S5). The linear organization of mammalian FAS domains annotated by Maier et. al., 2008, is shown in Fig. 3 (1). Conservation in linear organization of motifs associated with known functional domains identified using Pfam 31.0 software is shown in Fig. 3B. Dotted lines between Fig. 3A and B compare mammalian FAS domains (Fig. 3A) and AaFAS domains (Fig. 3B). Pfam analysis did not show the presence of functional methyltransferase domains in AaFAS (Fig. 3B) and protein sequence alignment using Clustal Omega showed deletion within pseudo-methyltransferase ( $\Psi$ ME) domains of AaFAS compared to human FAS (16.20-23.03\% identity; Table S5 and Fig. S2).

The gene model of AaFAS-like was 800 amino acids in length and contained all functional catalytic motifs, whereas AaFAS6 was 1,386 amino acids in length, and lacked catalytic motifs but contained the conserved 3' motif YKELRLRGY conserved in FAS (Fig. S1). AaFAS-like contains ketoacyl synthase, ketoacyl 
synthase_C and ketoacyl-synthase C-terminal extension domains, the first $5^{\prime}$ domains of AaFAS1-5 and human FAS (Fig. 3B), while AaFAS6 contains ADH zinc, $\beta$-ketoreductase, PP binding and thioesterase domains, the last four domains located 3' in AaFAS1-5 and human FAS (Fig. 3B). In the AaegL5 assembly, AaFAS-like and 6 are located on chromosome 2 at positions $429280401-429282870$ and $429275401-429279876$, respectively. It is possible that AaFAS-like and -6 reflect an error in genome assembly, or a gene duplication. However, molecular data and the inability to detect transcripts associated with either locus, suggest (Fig. S3) that AaFAS-like and 6 represent pseudogenes (Fig. 3B).

\section{FAS expression during Ae. aegypti development}

Mosquitoes undergo four developmental stages: egg, larva, pupa and adult. RT-PCR was used to explore the hypothesis that expression patterns of $A a F A S$ genes vary among these stages. Five individual mosquitoes were collected for each of the 4th larval instar, pupa and adult stages. Expression of each of the AaFAS genes was normalized to the average expression of the $\beta$-actin gene ( $\left.2^{-\Delta C t}\right)$ (Fig. 4$)$.

Relative expression analyses revealed negligible AaFAS expression in larval and pupal stages, while the highest expression of all genes except AaFAS4 were observed in adult males (Fig. 4). AaFAS1 was the most predominant FAS expressed in adult mosquitoes. Differences in expression levels of any AaFAS were not observed between sugar-fed and 3 days post blood-fed (coinciding with the first gonotrophic cycle) females. The study also revealed negligible AaFAS 4 expression in all developmental stages and sexes (Fig. 4).

\section{Impact of blood feeding on expression of AaFAS1}

The diet of the female Ae. aegypti typically involves both nectar and blood. The blood meal is rich in proteins and lipids; therefore, this diet may trigger lipolysis, instead of synthesis, to break down lipid molecules. We compared AaFAS1 expression, the predominant AaFAS in adult females, in sugar-fed females versus blood-fed females (feeding once or twice) (Fig. 5). Blood meals were provided only on specific days as shown in Fig. 5A, while mosquitoes from all groups were fed ad lib on $10 \%$ sugar diet throughout the experiment. Comparisons of AaFAS1 gene expression from mosquito samples collected on the same day showed no differences among feeding conditions (Fig. 5B). However, when profiled as ratios (Fig. 5C), we observed a slight, but not significant, reduction of AaFAS1 expression in females given a single blood-meal as compared to sugar-fed females on days 1, 3 and 4 post-blood meal (pbm) (Fig. 5B: $\mathrm{F}$ vs. B, G vs. $\mathrm{C}$ and $\mathrm{H}$ vs. D). This data suggests that diet may only play a minor role, if any, in the expression of AaFAS1 gene.

\section{Transient knockdown of AaFAS1 gene causing upregulation of other AaFAS genes}

We hypothesized that the redundancy of AaFAS genes may serve as a backup system for the mosquitoes. To test this hypothesis, we employed AaFAS1 lossof-function studies to investigate the possibility of compensation by other AaFAS genes. Female mosquitoes were IT injected with dsRNA derived from AaFAS1 or GFP (KD control). On day 2 post-dsRNA injection, five mosquitoes were collected for assessment of AaFAS expression (Fig. 6). We observed an approximate 40\% reduction in AaFAS1 expression ( 39.3 13.9\%) in AaFAS1-KD mosquitoes compared to the GFP-KD control (Fig. 6A). In AaFAS1-KD mosquitoes, expression levels of AaFAS2, 3 and 5 and were $191.7 \pm 38.6 \%, 161.4 \pm 21.8 \%$, and $191.1 \pm 38.9 \%$, respectively, in comparison to their levels in GFP-KD control, indicating possible compensation for the loss of AaFAS1 transcript. Conversely, the expression of $A a F A S 4$ was $87.71 \pm 74.0 \%$ compared to $A a F A S 4$ expression in GFP-KD control mosquitoes. To determine whether the upregulation observed in AaFAS2, 3, and 5 could possibly compensate for the loss of AaFAS1 in the AaFAS1-KD mosquitoes, we normalized the level of AaFAS genes to $\beta$-actin. We observed modest expression of AaFAS transcripts (5.6 $1.44 \%$ for AaFAS2, $4.6 \pm 0.00 \%$ for AaFAS3, and $7.07 \pm 0.62 \%$ for AaFAS5 compared to $\beta$-actin), while these upregulation still did not match the remnent of $A a F A S 1$ expression after the KD effect (36.1 $\pm 11.6 \%)$. This data suggest that other AaFASs may not be able to serve as a backup system for AaFAS1, at least in adult female mosquitoes under transient KD condition.

\section{Effect of RNAi-induced AaFAS1 knockdown on DENV2 replication in Ae. aegypti cells}

Studies in cell culture have shown that FAS activity is required for flavivirus genome replication $(13,14,37)$. Biochemical inhibition of FAS activity reduced DENV2 replication in both human and mosquito $C 6 / 36$ cells $(13,15,16)$. The lack of functional RNAi machinery in C6/36 cells hindered the use of transient KD strategy in mosquito cells. However, Ae. aegypti cells, Aag2, have functional RNAi machinery; therefore, we can investigate the role of AaFAS1, the most abundant transcript in female mosquitoes, in DENV2 replication using dsRNA transient KD in these cells (38). At 48 hours post-AaFAS1-KD (time zero of DENV2 infection), the expression level of AaFAS1 in Aag2 cells was $5.15 \pm 6.33 \%$ as compared to AaFAS1 expression in GFP-KD control cells (Fig. 7A). At 24 hours post DENV2 infection (72 hours post-KD), we observed significant reduction $(p<0.001)$ in DENV2 RNA replication in AaFAS1-KD cells as compared to the GFP-KD controls, comparable to replication in DENV2-KD (KD positive control) (Fig. 7B). KD was not associated with detrimental effects to the cells (Fig. 7C), suggesting that $A a F A S 1$ is required for DENV2 replication in mosquito cells.

\section{Transient inhibition of AaFAS1 reduced DENV2 infection in the midgut of Ae. aegypti}

To investigate the role of $A a F A S 1$ in DENV2 replication in vivo, mosquitoes were IT injected with dsRNA derived from AaFAS1 or GFP genes, and subsequently exposed to DENV2 infectious blood meal two-days post-injection (Fig. 8). On days 0, 3 and 7 pbm (corresponding to 2, 5 and 9 days post-dsRNA injection), whole mosquitoes were collected and analyzed for AaFAS1 gene expression (Fig. 8A). On the day of DENV2 infection by blood meal (2 days post-dsRNA injection), the level of $A a F A S 1$ expression was downregulated by $53.73 \pm 27.13 \%$ relative to GFP-KD group. On day 3 pbm, AaFAS1 expression recovered to $119.82 \pm 49.43 \%$ and was comparable to the AaFAS1 expression level in the GFP-KD control. On day 7 pbm, AaFAS1 was upregulated to $191.69 \pm 50.17 \%$, suggesting a possible over-compensation post KD effect (Fig. 8A).

Investigation of DENV2-fed mosquitoes showed that, at day $3 \mathrm{pbm}$, we observed significant reduction in percent of DENV2 infected midguts compared to the GFP-KD control (Fig. 8B). The odds ratio for AaFAS1 in AaFAS1-KD mosquitoes compared to GFP-KD mosquitoes on day 3 pbm was 0.20 (95\% confidence interval (CI): $0.06-0.60)$ and for DENV2 compared to GFP-KD was $0.03(\mathrm{Cl}: 0.01-0.13)$ suggesting fewer mosquitoes were infected with DENV2 in the 
AaFAS1-KD group compared to the GFP-KD group on day 3 pbm. However, no differences in percent infection were observed between AaFAS1-KD and control mosquitoes on days 7 and 14 pbm. Infectious particles produced from midguts (virus titer) from AaFAS1-KD and DENV2-KD groups were significantly different from GFP-KD group when the uninfected samples were included in the analysis using the nonparametric Kruskal-Wallis test followed by Dunn's test, with $\mathrm{p}$-values adjusted with the Bonferroni method $(p=0.0002$ and $p<0.0001$, respectively). However, if the titers of uninfected midguts were excluded, differences in virus titer among different dsRNA treatments were not detected (tested by one-way ANOVA followed by Dunn's test; virus titer AaFAS1-KD: 2.40 $\times 10^{2}$, GFP-KD: $2.18 \times 10^{3}$, and DENV2-KD: $6.43 \times 10^{2}$ plaque forming unit (PFU/midgut).

The inhibitory effect of AaFAS1-KD on DENV2 infection did not persist in the midgut beyond day $3 \mathrm{pbm}$. The titer and percent infection in the AaFAS1-KD mosquitoes were comparable to the GFP-KD mosquitoes on day 7 pbm (AaFAS1-KD: 2.70x10 ${ }^{3}$, GFP-KD: $2.95 \times 10^{4}$ and DENV2-KD: 5.88x10 ${ }^{3}$ PFU/midgut; Fig. 8 C). No differences in viral titer and percent infection were observed in midgut on day $7 \mathrm{pbm}$. To investigate whether transient $A a F A S 1-K D$ could disrupt virus dissemination, mosquito carcasses (whole body without midgut) were tested on day 14 pbm for virus infection (AaFAS1-KD: 6.57x10 ${ }^{4}$, GFP-KD: 8.47x10 4 and DENV2-KD: 0.00 PFU/carcass; Fig. 8D). Although we observed no statistical differences in mean titer in AaFAS1-KD as compared to GFP-KD control samples, two distinct populations of mosquitoes with viral titers in AaFAS1-KD carcasses were observed (Fig. 8D); some with viral titers comparable to GFP-

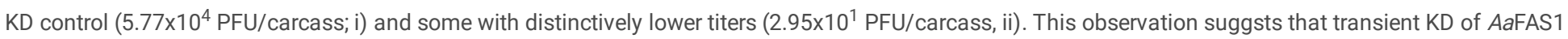
had a prolonged effect that can impact dissemination of DENV2 in mosquitoes.

\section{Discussion}

Lipids are essential for a variety of physiological processes in mosquitoes $(3,10,12,39,40)$. Mosquitoes not only acquire lipids from maternal (i.e. deposition to eggs) and dietary sources, but they also have the ability to synthesize lipids de novo. In this study, we characterized the expression of the AaFAS gene family, the key enzyme in the de novo lipid biosynthesis pathway. Additionally, we characterized the response of AaFAS1 expression during infection of mosquitoes and mosquito cell lines with DENV2 to investigate the potential role of this enzyme in supporting virus replication in the mosquito vector.

Seven putative AaFAS genes (AaFAS1-6 and AaFAS-like), were identified in the AaegL5 assembly based on amino acid similarity to FAS from vertebrates, invertebrates and yeast. Amino acid sequence alignments revealed low amino acid similarity $(<50 \%)$ of $A a F A S$ compared to human FAS (Table 1$)$.

The AaFAS1-5 possess seven catalytic and two of the three noncatalytic domains identified in the human FAS annotated using Pfam (Fig. 3) (1, 29). All AaFAS lack the UME domain observed in Drosophila melanogaster (fruit fly), Bombyx mori (silkworm), Apis mellifera (honey bee), Culex pipiens, and Anopheles gambiae (1). Similarly, mammalian FAS also loses the conservation of the motif involved in S-adenosyl-methionine (SAM)-dependent methyltransferases from the $\Psi \mathrm{ME}$, resulting in an absencce of methyltransferase activity, while it is highly conserved in bacteria and fungi (1). An absence of $\Psi \mathrm{ME}$ domain in insect FAS may reflect the unnecessity of this domain.

Gene duplication is a hallmark of many mosquito gene families and has been proposed as a source of new evolutionary features (23, 36, 41). Retention of duplicated genes may be indicative of positive/neutral selection and loci associated with a fitness advantage for the mosquito (42). We performed molecular and prelimnary functional characterization of the AaFAS gene family. We detected transcripts for five of the seven AaFAS genes. We were unable to detect transcripts for either AaFAS6 and AaFAS-like, and conclude that these gene models likely represent pseudogenes or may reflect an issue in the assembly.

Since mosquitoes undergo four distinct developmental stages in their life and these stages possess very distinct habitats and food sources, different $A a F A S$ genes may play roles supporting the unique requirements for FAS in these different life stages. Transcription profiles of $A a F A S 1-5$ revealed expression pattern. Notably, we observed low expression levels for all AaFAS in larval and pupal stages, suggesting that these genes may not be constitutively active across the mosquito life-cycle. We speculate that maternal lipids deposited in eggs during oogenesis (these comprise about $35 \%$ of dry egg weight (7)) and larvae diets may serve to support the metabolic needs during these stages $(8,9,43)$. Thus, they may have minimal requirement for de novo fatty acid biosynthesis.

We observed high levels of expression of all AaFAS, except AaFAS4, in males and to a lesser extent in females. Male Ae. aegypti do not blood feed, but solely obtain their diet from plant nectar, honeydew and fruits (44). Since these diets are high in carbohydrate but low in lipid content, high expression of $A a F A S$ in male mosquitoes may reflect a dependency on AaFAS for de novo synthesis of lipids.

Expression analyses also revealed AaFAS1 as the dominant AaFAS transcript in both male and female mosquitoes (Fig. 4). The AaFAS1 had the highst amino acid similarity among the AaFAS to the human and mouse FAS genes and is the putative paralogue of these genes. Upon AaFAS1-KD in female mosquitoes, we observed an $~ 2$-fold increase in other AaFAS transcripts indicating the attempt to compensate for the loss of AaFAS1 expression (Fig. 6A). Although we concluded that they fail to compensation for the loss of AaFAS1 since the expression levels of these AaFAS transcripts are still lower than the remaining AaFAS1 expression post-KD, improving the KD efficiency or extending the period of KD of AaFAS1 (such as using CRISPR-CAS9 knockout) may provide further insights into the redundancy of these AaFAS genes.

Since previous studies have demonstrated the importance of FAS activity in flavivirus replication in both human and mosquito cells $(13,15,16)$, we wanted to investigate if AaFAS1, also played an important role in DENV2 infection in the mosquito vector. Indeed, KD of AaFAS1 showed significant inhibition of DENV2 infection in midguts of AaFAS1-KD mosquitoes; however, this effect was only observed on day 3 pbm. This phenomenon might be caused by the transient KD of AaFAS expression. Alternative studies with longer suppression of AaFAS1 expression would be required to demonstrate the prominent impact on infection and for further transmission studies.

Nonetheless, we found an upregulation of AaFAS1 expression ( $200 \%$ increase compared to the AaFAS1 levels in the GFP-KD control) on day 9 post-KD. With relevance for strategies aimed at suppression of host factors for disrupting pathogen transmission,further studies are needed to better understand the biological impact of this "rebound" effect. 
Additionally, we observed a separation of the virus titers into two groups: high (Fig. 8D, i) and low (Fig. 8D, ii), titers in the carcass of the AaFAS1-KD mosquitoes. Ye et. al. 2015 , have shown that when mosquitoes were IT injected with DENV at $10^{6}$ PFU, they expectorated DENV into the saliva at about $10^{2}$ PFU, which is about 100-fold less than mosquitoes that were IT injected with DENV at $10^{7}$ PFU (approximate DENV titer in saliva $=10^{4} \mathrm{PFU}$ ) (45). This result suggests that viral titer of DENV in saliva may be dependent on the titer in the body (disseminated titers). In our observations, it is possible that mosquitoes with low body titers (group ii) would inefficienty transmit the virus and the KD of AaFAS1 may result in a reduction of transmission potential. This study suggests that biological relevance of low viral titres in carcasses and its impact on transmission dynamics is worthy of further investigation.

\section{Conclusions}

Here we pesent expression analyses of the AaFAS gene family and a focused study of the AaFAS1 in Ae. aegypti. We annotated seven AaFAS genes from the AaegL5 genome assembly, and present evidence to support function of five genes. Expression data revealed complexities of $A a F A S$ expression between stages and sexes, and suggest that AaFAS1 is the dominant transcript in both male and female adult mossquitoes. Sequence homology suggested conservation between mammalian FAS and AaFAS1, and the presence of multiple catalytic domains supports AaFAS1 as a key enzyme in de novo lipid biosynthesis. In addition, AaFAS1 was found to facilitate DENV2 replication in both cell culture and in Ae. aegypti. In the latter case, it demonstrated the potential to affect vector competency for virus transmission.

\section{Abbreviations}

ACC; acetyl-CoA carboxylase,Ae.aegypti: Aedes aegypti; AaFAS: Aedes aegyptifatty acid synthase; bp: base pair; cDNA: complementary deoxyribonucleic acid; DENV2: dengue virus serotype 2; dpi: days post-infection; dsRNA: long double-stranded ribonucleic acid; FAS: fatty acid synthase; FBS: fetal bovine serum; GFP: green fluorescent protein; IT: intrathoracic; KD: knockdown; MEM: Minimum Essential Media; PBS: phosphate-buffered saline; mRNA: messenger ribonucleic acid; PCR: polymerase chain reaction; quantitative polymerase chain reaction; pbm: post-blood meal; PFU: plaque forming unit; YME: pseudomethyltransferase;RNA, ribonucleic acid; RNAi: interference ribonucleic acid; RT: reverse transcription; RT-PCR: reverse transcriptionpolymerase chain reaction; SNP: single nucleotide polymorphism

\section{Declarations}

\section{Acknowledgements}

The authors thank Kenneth E. Olson and Irma Sanchez-Vargas for providing mosquito eggs. We also thank Rebecca Gullberg, Laura St. Clair , Jeffrey M. Grabowski and Richard J. Kuhn for advice on experimental design, data analysis and critical evaluation of the manuscript and Tach Costello for clerical and administrative support. Graphical Abstract was created using BioRender.

\section{Funding}

This work was funded by R01Al151166 NIH-NIAID and the Boettcher Foundation Early Career Investigator Awards to RP. GR was funded by the R01Al151166 NIH-NIAID. CBS was supported by Purdue internal monies.

Supplement.

\section{Availability of Data and Materials}

All data and materials were presented in the manuscript and supplementary information.

\section{Authors' contribution}

Author Contributions:NC, CBS, GR and EL carried out theexperiments. NC, CBS, GR, EL, CAH and RP wrote themanuscript.

\section{Ethics approval and consent to participate}

Not applicable

\section{Consent for publication}

All authors consent for publication

\section{Competing interest}

The authors have no competing interests.

\section{References}

1. Maier T, Leibundgut M, Ban N. The crystal structure of a mammalian fatty acid synthase. Science. 2008;321(5894):1315-22.

2. White SW, Zheng J, Zhang YM, Rock. The structural biology of type II fatty acid biosynthesis. Annu Rev Biochem. 2005;74:791-831.

3. Arrese EL, Soulages JL. Insect fat body: energy, metabolism, and regulation. Annu Rev Entomol. 2010;55:207-25. 
4. Dadd RH, Kleinjan JE. Essential fatty acid for the mosquito Culex pipiens: arachidonic acid. J Insect Physiol. 1979;25(6):495-502.

5. Stanley DW, Miller JS. Eicosanoid actions in insect cellular immune functions. Entomologia Experimentalis Et Applicata. 2006;119(1):1-13.

6. Ziegler R. Lipid synthesis by overies and fat body of Aedes aegypti (Diptera: Culicidae). European Journal of Entomology. 1997;94:385-91.

7. Ziegler R, Ibrahim MM. Formation of lipid reserves in fat body and eggs of the yellow fever mosquito, Aedes aegypti. Journal of Insect Physiology. 2001;47(6):623-7.

8. Atella GC, Shahabuddin M. Differential partitioning of maternal fatty acid and phospholipid in neonate mosquito larvae. J Exp Biol. 2002;205(Pt 23):362330 .

9. Sushchik NN, Yurchenko YA, Gladyshev MI, Belevich OE, Kalachova GS, Kolmakova AA. Comparison of fatty acid contents and composition in major lipid classes of larvae and adults of mosquitoes (Diptera: Culicidae) from a steppe region. Insect Sci. 2013;20(5):585-600.

10. Zhou GL, Pennington JE, Wells MA. Utilization of pre-existing energy stores of female Aedes aegypti mosquitoes during the first gonotrophic cycle. Insect Biochemistry and Molecular Biology. 2004;34(9):919-25.

11. Jenkin HM, McMeans E, Anderson LE, Yang TK. Comparison of phospholipid composition of Aedes aegypti and Aedes albopictus cells obtained from logarithmic and stationary phases of growth. Lipids. 1975;10(11):686-94.

12. Alabaster A, Isoe J, Zhou G, Lee A, Murphy A, Day WA, et al. Deficiencies in acetyl-CoA carboxylase and fatty acid synthase 1 differentially affect eggshell formation and blood meal digestion in Aedes aegypti. Insect Biochem Mol Biol. 2011;41(12):946-55.

13. Heaton NS, Perera R, Berger KL, Khadka S, Lacount DJ, Kuhn RJ, et al. Dengue virus nonstructural protein 3 redistributes fatty acid synthase to sites of viral replication and increases cellular fatty acid synthesis. Proc Natl Acad Sci U S A. 2010;107(40):17345-50.

14. Martín-Acebes M, Blázquez A, Oya NJd, Escribano-Romero E, Saiz J. West Nile virus replication requires fatty acid synthesis but is independent on phosphatidylinositol-4-phosphate lipids. Plos One. 2011;6(9).

15. Perera R, Riley C, Isaac G, Hopf-Jannasch AS, Moore RJ, Weitz KW, et al. Dengue virus infection perturbs lipid homeostasis in infected mosquito cells. PLoS Pathog. 2012;8(3):e1002584.

16. Tongluan N, Ramphan S, Wintachai P, Jaresitthikunchai J, Khongwichit S, Wikan N, et al. Involvement of fatty acid synthase in dengue virus infection. Virol J. 2017;14(1):28.

17. Welsch S, Miller S, Romero-Brey I, Merz A, Bleck CK, Walther P, et al. Composition and three-dimensional architecture of the dengue virus replication and assembly sites. Cell Host Microbe. 2009;5(4):365-75.

18. Junjhon J, Pennington JG, Edwards TJ, Perera R, Lanman J, Kuhn RJ. Ultrastructural characterization and three-dimensional architecture of replication sites in dengue virus-infected mosquito cells. J Virol. 2014;88(9):4687-97.

19. Harak C, Lohmann V. Ultrastructure of the replication sites of positive-strand RNA viruses. Virology. 2015;479-480:418-33.

20. Chotiwan N, Andre BG, Sanchez-Vargas I, Islam MN, Grabowski JM, Hopf-Jannasch A, et al. Dynamic remodeling of lipids coincides with dengue virus replication in the midgut of Aedes aegypti mosquitoes. PLoS Pathog. 2018;14(2):e1006853.

21. Camacho C, Coulouris G, Avagyan V, Ma N, Papadopoulos J, Bealer K, et al. BLAST+: architecture and applications. BMC Bioinformatics. $2009 ; 10: 421$.

22. Giraldo-Calderon GI, Emrich SJ, MacCallum RM, Maslen G, Dialynas E, Topalis P, et al. VectorBase: an updated bioinformatics resource for invertebrate vectors and other organisms related with human diseases. Nucleic Acids Res. 2015;43(Database issue):D707-13.

23. Nene V, Wortman JR, Lawson D, Haas B, Kodira C, Tu ZJ, et al. Genome sequence of Aedes aegypti, a major arbovirus vector. Science. 2007;316(5832):1718-23.

24. Larkin MA, Blackshields G, Brown NP, Chenna R, McGettigan PA, McWilliam H, et al. Clustal W and Clustal X version 2.0. Bioinformatics. 2007;23(21):2947-8.

25. Corpet F. Multiple sequence alignment with hierarchical clustering. Nucleic Acids Res. 1988;16(22):10881-90.

26. Beedessee G, Hisata K, Roy MC, Satoh N, Shoguchi E. Multifunctional polyketide synthase genes identified by genomic survey of the symbiotic dinoflagellate, Symbiodinium minutum. BMC Genomics. 2015;16:941

27. Sievers F, Wilm A, Dineen D, Gibson TJ, Karplus K, Li W, et al. Fast, scalable generation of high-quality protein multiple sequence alignments using Clustal Omega. Mol Syst Biol. 2011;7:539.

28. Brown NP, Leroy C, Sander C. MView: a web-compatible database search or multiple alignment viewer. Bioinformatics. 1998;14(4):380-1.

29. Mistry J, Bateman A, Finn RD. Predicting active site residue annotations in the Pfam database. BMC Bioinformatics. 2007;8:298.

30. Bennett KE, Olson KE, Munoz Mde L, Fernandez-Salas I, Farfan-Ale JA, Higgs S, et al. Variation in vector competence for dengue 2 virus among 24 collections of Aedes aegypti from Mexico and the United States. Am J Trop Med Hyg. 2002;67(1):85-92.

31. Mathur G, Sanchez-Vargas I, Alvarez D, Olson KE, Marinotti O, James AA. Transgene-mediated suppression of dengue viruses in the salivary glands of the yellow fever mosquito, Aedes aegypti. Insect Mol Biol. 2010;19(6):753-63.

32. Schmittgen TD, Livak KJ. Analyzing real-time PCR data by the comparative C(T) method. Nat Protoc. 2008;3(6):1101-8.

33. Deubel V, Kinney RM, Trent DW. Nucleotide sequence and deduced amino acid sequence of the structural proteins of dengue type 2 virus, Jamaica genotype. Virology. 1986;155(2):365-77.

34. Merrill AH, Jr., Sullards MC, Allegood JC, Kelly S, Wang E. Sphingolipidomics: high-throughput, structure-specific, and quantitative analysis of sphingolipids by liquid chromatography tandem mass spectrometry. Methods. 2005;36(2):207-24.

35. Lee E, Helt GA, Reese JT, Munoz-Torres MC, Childers CP, Buels RM, et al. Web Apollo: a web-based genomic annotation editing platform. Genome Biol. 2013;14(8):R93 
36. Matthews BJ, McBride CS, DeGennaro M, Despo O, Vosshall LB. The neurotranscriptome of the Aedes aegypti mosquito. BMC Genomics. $2016 ; 17: 32$.

37. Tang WC, Lin RJ, Liao CL, Lin YL. Rab18 facilitates dengue virus infection by targeting fatty acid synthase to sites of viral replication. J Virol. 2014;88(12):6793-804.

38. Sanchez-Vargas I, Scott JC, Poole-Smith BK, Franz AW, Barbosa-Solomieu V, Wilusz J, et al. Dengue virus type 2 infections of Aedes aegypti are modulated by the mosquito's RNA interference pathway. PLoS Pathog. 2009;5(2):e1000299.

39. Downer RGH, Matthews JR. Patterns of lipid distribution and utilisation in insect. American Zoologist. 1976;16(4):733-45.

40. Zhou GL, Flowers M, Friedrich K, Horton J, Pennington J, Wells MA. Metabolic fate of [C-14]-labeled meal protein amino acids in Aedes aegypti mosquitoes. Journal of Insect Physiology. 2004;50(4):337-49.

41. Chau LM, Goodisman MAD. Gene duplication and the evolution of phenotypic diversity in insect societies. Evolution. 2017;71(12):2871-84.

42. Helmkampf M, Cash E, Gadau J. Evolution of the insect desaturase gene family with an emphasis on social Hymenoptera. Mol Biol Evol. 2015;32(2):45671.

43. Dadd RH. Essential Fatty-Acids for the Mosquito Culex-Pipiens. J Nutr. 1980;110(6):1152-60.

44. Wahid I, Sunahara T, Mogi M. Maxillae and mandibles of male mosquitoes and female autogenous mosquitoes (Diptera: Culicidae). J Med Entomol. 2003;40(2):150-8.

45. Ye YH, Carrasco AM, Frentiu FD, Chenoweth SF, Beebe NW, van den Hurk AF, et al. Wolbachia Reduces the Transmission Potential of Dengue-Infected Aedes aegypti. PLoS Negl Trop Dis. 2015;9(6):e0003894.

\section{Figures}

\section{Figure 1}

Schematic showing the predicted gene structure of the AaFAS gene family. Exons are indicated by yellow cylindrical bars, 5' and 3' non-coding exons by dark grey shading, introns by a black line, start codon by green arrow and stop codon by red T.

\section{Figure 2}

Phylogenetic analysis of AaFAS. Bayesian phylogeny represented with an unrooted tree showing the main relationships between mosquito FAS genes and their counterparts in Ag: Anopheles gambiae, Dm: Drosophila melanogaster, Am: Apis mellifera, Mm: Mus musculus, Hm: Homo sapiens and Sc: Saccharomyces cerevisiae. The branches are supported by posterior probability values. The tree is drawn to scale: amino acid changes per site.

\section{Figure 3}

Linear organization of Aedes aegypti FAS genes showing functional domains. (a) Schematic shows linear organization of 7 catalytic and 3 noncatalytic domains of mammalian FAS annotated by Maier et. al., 2008(1). Seven catalytic domains are shown in big squares and 3 non-catalytic domains are shown in smaller squares. Abbreviations: KS, b-ketoacyl synthase; LD, linker; MAT, malonyl-acetyl transferase; DH, dehydratase; YME, pseudo-methyl transferase; YKR, pseudo b-ketoacyl synthase; ER, enoyl reductase; KR, b-ketoacyl synthase; ACP; acyl carrier protein and TE, thioesterase. (b) Schematics show conserved domains or motifs of FAS genes and their organization annotated using Pfam 31.0 software. Abbreviations are as follows: ketoacyl_synt, b-ketoacyl synthase; ketoacyl_synt_C, b-ketoacyl-acyl carrier protein synthase; ketoacyl_assoc, ketoacyl-synthase C-terminal extension; acyl_transf_1, acyl transferase domain; PS$\mathrm{DH}$, polyketide synthase; methyltransf, methyltransferase domain; ADH-zinc, zinc binding dehydrogenase; KR, b-ketoreductase domain; PP-binding, phosphopantetheine attachment site; thioesterase, thioesterase domain.

\section{Figure 4}

Expression of AaFAS in mosquito developmental stages and sexes. RNA was prepared from 5 specimens of $4^{\text {th }}$ instar larvae, pupae, sugar-fed males, sugarfed females, and females 3-days pbm (all adult mosquitoes were collected at the same day time; 8-10 days post eclosion). Samples were subjected to RTqPCR to assess relative expression of AaFAS1-5 and AaFAS-like. RNA levels between samples were normalized to the b-actin gene using $2^{-\mathrm{DCt}}$ method. The boxes show the $25^{\text {th }}$ and $75^{\text {th }}$ percentiles, the whiskers show the minimum and maximum values and the midline indicates the median of the relative gene expression value.

\section{Figure 5}


Expression of AaFAS1 in sugar-fed and blood-fed mosquitoes. (a) Schematic of the experimental design. Mosquitoes were reared in 3 different conditions: sugar-fed only, one blood meal, which were fed on day zero, and two blood meals, which were again fed on day 3. Mosquitoes in all conditions were also allowed access to additional sugar and water at all time throughout the experiment. Five mosquitoes from each condition were collected on different days post $1^{\text {st }}$ blood meal feeding designated in letters A-K. (b) AaFAS1 expression profile (2 $2^{\text {-DCt}}$; normalized to actin) of each sampling group were shown. Statistical analysis was performed by one-way ANOVA followed by Tukey's multiple comparison tests. ${ }^{*}, \mathrm{p}<0.05$ and $* *$, $p<0.01$. (c) Expression of AaFAS1 was measured by the comparative expression $\left(2^{-D D C t}\right)$ method. The samples used for comparisons are shown on the X-axis. Boxes show the $25^{\text {th }}$ and $75^{\text {th }}$ percentiles, whiskers show the minimum and maximum values, and the midline shows the median of the relative gene expression value.

\section{Figure 6}

Comparive analyses of AaFAS expression in AaFAS1-KD mosquito. Three-day-old adult female mosquitoes were intrathoracically injected with dsRNA derived from AaFAS1 or GFP mRNA sequence (an irrelevant dsRNA control). Mosquitoes were reared on 10\% sugar diet for 2 days post-injection. Three pools of 5 mosquitoes were collected for AaFAS gene expression measurements. (a) Percent relative expression of AaFAS genes in AaFAS1-KD mosquitoes was compared to GFP-KD control mosquitoes using the comparative Ct (2-DDCt) method. (b) Gene expression profiles of AaFASwere measured as normalized to bactin gene expression $\left(2^{-D C t}\right)$. Boxes show the $25^{\text {th }}$ and $75^{\text {th }}$ percentiles, whiskers show the minimum and maximum values and midline shows median of the relative gene expression value. Statistical analysis was performed by one-way ANOVA followed by Tukey's multiple comparison tests. ${ }^{\star *}, p<0.01,{ }^{\star \star \star}, p<0.005$ and $* * *, p<0.001$.

\section{Figure 7}

Assessment of RNAi-induced AaFAS1 knockdown on DENV2 replication in Aag2 cells. (a) Percent AaFAS1 expression in AaFAS1-KD Aag2 cells compared to the AaFAS1 expression in GFP-KD cells. Expression was measured at two days post dsRNA transfection. (b) RNA replication of luciferase-expressing DENV2 in Aag2 cells treated with dsRNA derived from AaFAS1, DENV2 or GFP. Cells were transfected with dsRNA for two days prior to infection with luciferase-tagged DENV2. At $24 \mathrm{hpi}$, cells were lysed and were assayed for luciferase activity (RLU). One-way ANOVA followed by Tukey's multiple comparison test were applied for statistical analysis. ${ }^{* \star *}, \mathrm{p}<0.001$. (c) Viability of Aag2 cells treated with dsRNA derived from AaFAS1, DENV2 (positive DENV2 KD control) and GFP (negative DENV2 KD control) genes assessed by resazurin assay.

\section{Figure 8}

Transient KD of AaFAS1 expression by dsRNA temporarily reduced DENV2 infection in midguts. (a) Percent $A a F A S 1$ expression in FAS-KD mosquitoes. Mosquitoes were IT injected with $400 \mathrm{ng}$ of dsRNA derived from AaFAS1, DENV2 or GFP two days prior to blood meal infection. On days 0,3 and 7 pbm (days 2, 5 and 9 days post IT injection), 3 pools of 5 mosquitoes were collected and analyzed for AaFAS1 expression compared to AaFAS1 expression in GFPKD mosquitoes. (b-d) Mosquitoes were IT injected with dsRNAs and infected with DENV2 by infectious blood meal at 2 days post injection. Plaque assay was performed on midguts dissected on (b) day 3 and (c) day 7 and (d) carcasses (whole body without midgut) collected on day 14 pbm. (i) and (ii) indicate the separation of DENV2 titers in the carcass that were produced from the AaFAS1-KD mosquitoes.Mean virus titer (infectious particles) was calculated for infected samples only. One-way ANOVA followed by Dunn's tests were applied to test the differences in virus titer among samples, *, $p<0.05$. The odds ratio was applied to test the differences in percent infection. The significant reduction of percent infection in AaFAS1-KD or DENV2-KD compared to GFP-KD are highlighted in bold.

\section{Supplementary Files}

This is a list of supplementary files associated with this preprint. Click to download.

- Additionalfile1Chotiwanetal.docx

- Additionalfile2Chotiwanetal.docx

- Figures1Chotiwanetal.tiff

- Figures2Chotiwanetal.tiff

- Figures3Chotiwanetal.tiff

- Graphicalabstract.tiff 